\title{
Detection of Interleukin 6 (IL-6) antigen in the head and neck carcinoma cells
}

\author{
Július Rajčáni ${ }^{1 *}$, Ferenc Bánáti ${ }^{1}$, Kálmán Szenthe ${ }^{1}$, Hans Helmut Niller ${ }^{2}$, János Minarovits ${ }^{3}$, László Stipkovits ${ }^{4}$ and Susan Szathmary $^{5}$ \\ ${ }^{1}$ RT-Europe Nonprofit Research Center, Mosonmagyaróvár, Hungary \\ ${ }^{2}$ Institute of Medical Microbiology and Hygiene, University of Regensburg, Regensburg, Germany \\ ${ }^{3}$ University of Szeged, Faculty of Dentistry, Department of Oral Biology and Experimental Dental Research, Hungary \\ ${ }^{4}$ Carlsbad Research Org. Ltd, Mosonmagyaróvár, Hungary \\ ${ }^{5}$ Galenbio Ltd, Mosonmagyaróvár, Hungary
}

\begin{abstract}
Background: Interleukin 6 (IL-6) is a pleiotropic cytokine produced not only by inflammatory cells, but also by the squamous epithelium of oral cavity, tongue and/ or laryngeal mucosa (probably explaining its presence in saliva and respiratory secretions).

Methods: Immunohistochemical staining for IL-6 antigen was performed in histological sections coming from 98 head and neck squamous cell carcinoma (HNSCC) samples using monospecific IL-6 antibody (rabbit anti-IL-6 serum purchased from Abcam, product Ab6672) and taking advantage of the BenchMark Ultra IHC/ ISH staining module (Ventana Discovery XT automated system, Tuscon, USA).

Results: The expression of IL-6 antigen was examined in a total of the 98 head and neck squamous cell carcinoma (HNSCC) samples, i.e. 33 laryngeal, 55 tongue and 10 nasal cavity specimens, along with 18 relevant normal tissue samples (negative controls) and, in addition, in 10 non-HNSCC tumours (alternative tumour controls). Immuhistochemical staining confirmed the presence of IL-6 antigen in the cytoplasm of carcinoma cells as well as in the stroma of all the HNSCC tumours (100\%). In conventional squamous carcinomas a rather confluent staining of IL-6 could be seen in the cytoplasm of dysplastic prickle cells, while in anaplastic cells of undifferentiated carcinomas a less intensive fine granular staining pattern dominated. For comparison, occasional and less intensive staining for the IL-6 antigen was seen in 5 out of 10 alternative carcinomas (50\%) and/or in 10 out of $16(60 \%)$ relevant normal tissue controls.
\end{abstract}

Conclusion: We confirmed the widespread expression IL-6 antigen as detected by immunohistochemical staining in all the HNSCC samples examined (100\% positive), regardless whether using purchased slides or sections prepared from our domestic biopsy material. A proportion (60 \%) of the normal laryngeal and mouth cavity epithelium was positive as well, though in the letter, the frequency and intensity of antigen expression was considerably lower.

\section{Introduction}

Interleukin 6 (IL-6) is a glycoprotein consisting of 184 amino acids, which has been first identified as a T-cell-derived regulatory factor controlling B cell differentiation, but more recently regarded for a multifunctional pleiotropic cytokine acting in a variety of cells [1] regulating immune responses, hematopoiesis, inflammation as well as production of acute-phase proteins by liver hepatocytes [2]. In addition to $\mathrm{T}$ cells, $\mathrm{B}$ cells and macrophages, production and secretion of IL-6 was described in adipocytes, in capillary endothelium cells as well as in activated connective tissue fibroblasts including the infiltrating immune cells. Several malignant tumours (such as multiple myeloma, lymphoma, ovarian cancer, prostate cancer, lung cancer, breast cancer, metastatic and renal carcinomas) may express IL-6, but most frequently, the head and neck squamous carcinoma (HNSCC) represents an abundant cytokine-producing neoplasm [3].

The IL-6 production can originate in cancer associated fibroblasts (CAFs) at immediate vicinity of the HN SCC tumour, and/or from mobile mesenchymal stem cells, which both may secrete various oncogenic cytokines. To become cancer associated fibroblasts (CAFs) the stroma cell undergoes a phenotype switch and start expressing various markers such as alpha-smooth muscle actin (б-SMA), fibroblast specific protein (FSP1), vimentin and periostatin [4]. Cytokine secretion into the tumour microenvironment may, on one hand, help immune defence to attack the tumour $[5,6]$, but on other hand, it may promote the proliferation of tumour cells when activating the signal transducer and activator of transcription 3 (STAT3) pathway contributing to tumour progression [7]. CAFs residing within the tumour stroma promote the spread of HNSCC, facilitating angiogenesis and overcoming the action of immune $\mathrm{T}$ cells [8]. At high concentrations wthin cancer tissue, IL-6 deregulates several signalling pathways influencing tumour cell invasion and metastasis $[9,10]$.

Elevated IL-6 levels present in the biopsy samples of HNSCC patients should be considered for a bad prognostic factor correlating with the worsening of clinical stage and a higher tumour recurrence rate, while a targeted curative treatment resulted in increased survival rate [11]. By means of the stroma derived factor-1 (SDF-1), the

*Correspondence to: Július Rajčáni, RT-Europe Nonprofit Research Center, Vár tér 2, Building E, HU-9920, Mosonmagyaróvár, Hungary, E-mail: viruraj@savba.sk

Key words: larynx, tongue, mouth and nose cavity tumours, squamous cell carcinoma, IL-6 expression, immunohistochemical staining

Received: February 05, 2019; Accepted: February 21, 2019; Published: February 28,2019 
STAT3 pathway is activated to become a central mediator implicated at tumour maintenance and/or progression [12,13]. Elevated levels of activated STAT3, phosphorylated at a single tyrosine residue (Y705) of its transactivation domain, are frequently detected in tumour biopsies, saliva and blood plasma [14-19]. When released by tumour cells, the IL-6 interacts with the soluble IL-6 receptor (sIL$6 \mathrm{R}$ ), an event conferring resistance against chemo- and radiotherapy treatments [20-22]. Furthermore, the overexpressed epidermal growth factor (EGF) can initiate transcription of the proliferation-associated genes (including STAT3) and activate related pathways "cooperating" with phospho-inositol-3 kinase (PI3K), since both transactivators function downstream of the EGF receptor (EGFR) [23,24]. Elevated concentrations of growth factors and cytokines (that activate the STAT3 pathway) have been indeed detected in the serum and/or tumour microenvironment of patients with HNSCC. The elevated IL-6 concentrations in the serum are markers of adverse prognosis, since the patients involved have been found associated with a higher stage of cancer development, faster tumour progression, inhibition of apoptosis, stimulation of angiogenesis as well as with drug resistance [25].

Plasma levels of Th2 cytokines along with tumour necrosis factor- $\alpha$ (TNF) and C-reactive protein appear to represent useful markers for early stages of tumour development [26-28]. In result of this, IL-6 is regarded for a valuable biomarker at estimating clinical prognosis [29-32], and for predicting the HNSCC recurrence [27] or calculating the patient's survival rate [34-37]. Our paper describes the frequency of IL- 6 production as detected by immunohistochemical stainig in 98 HNSCC samples among to confirm the abundant production of IL- 6 antigen by carcinoma cells as well as by fibrocytes in their stroma.

\section{Materials and methods}

\section{The origin of tumour samples}

A proportion of SCC sections was supplied by the head and neck tumour array (HN801a) [34], which consisted of 33 sections from laryngeal SCC specimens (at various differentiation grades and different growth stages) along with 5 normal laryngeal tissue samples, (together 36 laryngeal sections). Furthermore, the array provided 21 sections of tongue SCC specimens along with 10 control lingual epithelium samples. Finally, the array had 10 sections prepared from tumours of nasal and/or sinus cavities, along with 3 normal nasal mucosa samples coming from areas adjacent to tumour tissue. Taken together, the HN801a array provided 64 sections coming from HNSCC samples along with 16 relevant normal (control) tissue sections (altogether 80 sections, Table 1). Noteworthy that one additional section on the array was coming from a non-HNSCC tumour, namely from pheochromocytoma (a malignant adrenal gland tumour), representing the negative (IL-6 antigen free) control declared by the manufacturer. In addition to the array sections, 10 domestic carcinoma specimens of other than head and neck origin [26] (i.e. non-HNSCC tumours), were included into the study (3 these were bronchial carcinomas). In order to assess the possible influence of prolonged storage (under standard conditions) as well as the outcome of the embedding procedure on the intensity of IL- 6 antigen staining, additional 34 tongue and mouth cavity SCCs (i.e. also HNSCC tumours) were obtained from the collection of the Pathology Department of St. Elisabeth Oncology Hospital in Bratislava. The latter sections were coming from specimens removed for diagnostic purposes at curative surgery and were treated and stained in the same manner as the array sections (described below).

\section{Immunostaining}

The 5 micrometer $(\mathrm{Om})$ thick sections coming from the embedded HN SCC blocks (which origin has been described above) along with the sections on purchased array slides, were stained and processed in the BenchMark Ultra IHC/ISH staining module (Ventana Discovery XT automated system, Tuscon, USA). The protocol number 140 (at slightly acidic $\mathrm{pH}$ by an average of 6.2) and/or number 102 (at neutral $\mathrm{pH}$ of 7.2) were employed by using the universal $\mathrm{U}$-ultraview Red staining procedure and closely following the instructions of

Table 1. Survey of tissue samples examined for the IL-6 antigen

\begin{tabular}{|c|c|c|c|c|}
\hline Tissue of origin & Diagnosis & Positive out of total* & Sample source & Notice \\
\hline Larynx & $\mathrm{SCC}$ & $33 / 33(100 \%)$ & tissue array & $\begin{array}{l}\text { conventional SCC (19) } \\
\text { undifferentiated SCC (14) }\end{array}$ \\
\hline Larynx & normal tissue & $2 / 3(66 \%)$ & tissue array & \\
\hline Larynx samples total & & $35 / 36$ & & \\
\hline Tongue & $\mathrm{SCC}$ & $21 / 21(100 \%)$ & tissue array & $\begin{array}{l}\text { conventional SCC (9) } \\
\text { undifferentiated SCC (12) }\end{array}$ \\
\hline Tongue & $\mathrm{SCC}$ & $34 / 34(100 \%)$ & biopsy $^{1}$ & $\begin{array}{l}\text { conventional SCC (27) } \\
\text { undifferentiated SCC (7) }\end{array}$ \\
\hline Tongue & tumour tissue total & $55 / 55(100 \%)$ & & \\
\hline Tongue & normal tissue & $7 / 10(70 \%)$ & tissue array and biopsy ${ }^{1}$ & \\
\hline Tongue samples total & & $62 / 65$ & & \\
\hline Nose** & SCC & $10 / 10(100 \%)$ & tissue array & $\begin{array}{l}\text { conventional SCC (4) } \\
\text { undifferentiated SCC (6) }\end{array}$ \\
\hline Nose** & normal tissue & $1 / 3(33 \%)$ & array and biopsy ${ }^{1}$ & \\
\hline Nasal samples total & & $11 / 13$ & & \\
\hline HN samples total & SCC & 98/98 (100\%) & & \\
\hline HN samples total & normal tissue & $10 / 16(63 \%)$ & & \\
\hline All HN samples & & $108 / 114$ & & \\
\hline Non-HNSCC samples & various carcinomas $* * *$ & $5 / 10(50 \%) * * *$ & biopsy $^{2}$ & \\
\hline
\end{tabular}

*positive rate

$* *$ including paranasal sinuses

***Non-HNSCC tumours: uterine cervix, bronchial carcinoma, several GIT adenocarcinomas

${ }^{1}$ Biopsy from the Dept. of Pathology, St. Elisabeth Oncology Hospital, Bratislava, Slovakia

${ }^{2}$ Biopsy from Pathology Ltd, Alpha medical Company, Martin, Slovakia 
the manufacturer (Roche). The specificity of the IL-6 antibody (rabbit anti-IL-6 serum purchased from Abcam, product Ab6672) was checked by including the selected domestic SCC specimens (as listed on Table1). Alternative sections were stained for cytokeratin $5 / 6(\mathrm{CK} 5 / 6)$ with a commercial monospecific antibody. At first trial, the serum samples were diluted either 1:200 and/or 1:100 in the VA reaction buffer (provided by the manufacturer) and allowed to react under two different (neutral and moderate acidic $\mathrm{pH}$ ) processing conditions. Later on, for staining of the great majority of sections, the protocol number 140 (taking advantage of a slightly decreased $\mathrm{pH}$ [i.e. $<6.5$, but $>6.0]$ ) was used. This procedure (designated protocol 140A) was further modified (protocol 140B) by adding bovine serum albumin (BSA) at $2 \%$ concentration ( $200 \mathrm{mg}$ BSA $/ 100 \mathrm{ml}$ of VA diluent). Finally, when performing the negative staining control procedure $(C)$, all the reagents were similar as in the procedure A but omitting the specific anti-IL-6 serum only.

Briefly, the sections were deparaffinised at $72^{\circ} \mathrm{C}$ for $4 \mathrm{~min}$ in the EZ Prep solution provided by the kit, and then rehydrated. To unmask the IL- 6 antigen, all the sections were incubated at $96{ }^{\circ} \mathrm{C}$ for $8 \mathrm{~min}$ in the EZPrep CC solution provided. The repeatedly rinsed slides were immunostained with the polyclonal rabbit anti-IL- 6 antibody and/or the anti-CK5/6 antibody (both diluted in the VA reaction buffer at 1 : 200 and applied at $\mathrm{pH}<6.5$, at above referred to as procedure A). After incubation at $36^{\circ} \mathrm{C}$ for $1 \mathrm{hr}$ and repeated rinsing, the UV Red UNIV MULT reagent was applied on all the sections (for $12 \mathrm{~min}$ ), which were thereafter incubated in the presence of the UV RED Enhancer. The reaction was visualized by adding one drop of UV Fast Red and one drop of UV Red Naphthol (both were allowed to react for additional $8 \mathrm{~min}$ ). Finally, the sections were counterstained with hematoxylin (for $12 \mathrm{~min}$ ) and then handled with the Blueing reagent (for $12 \mathrm{~min}$ ). After repeated washing in the reaction buffer, the slides were mounted into a permanent medium when covered by a cover slip. All the stained sections were viewed in a Zeiss D1 observer microscope (Plan Apochromat objectives 10x, 20x and/or 40x) and photographed using the Zeiss Axiovision program.

\section{Standard histology}

Parallel sections from each block were also stained by the classical hematoxylin and eosin (HE) procedure.

\section{Results}

\section{Occurrence of IL-6 antigen in normal mouth, neck and nose} mucosa

Sections from 3 normal larynx samples, from 10 normal tongue samples and from 3 normal nasal mucosa samples, i.e. alltogether 16 normal head and neck mucosa samples, were stained for the presence of IL-6 antigen. Parallel control sections were treated similarly, but in the absence of specific antibody, while an additional series of sections was stained by standard, HE procedure. The normal squamous epithelium of at least 2 larynx samples showed groups of IL-6 positive prickle cells located mainly in the middle sheet of epithelial layer (Figure 1). A few fibrocytes in the underlying connective tissue were positive as well. The normal squamous epithelium was positive in 7 out of 10 lingual samples, namely the cytoplasm of prickle cells in the parabasal layer at proliferating areas in close vicinity of carcinoma (Figure 1). The underlying connective tissue was occasionally positive as well, especially the cytoplasm of a few fibrocytes. In addition, some extracellular antigen deposits were found showing an elongated shape resembling to collagen fibres. Faint positive staining for IL-6 was also found in

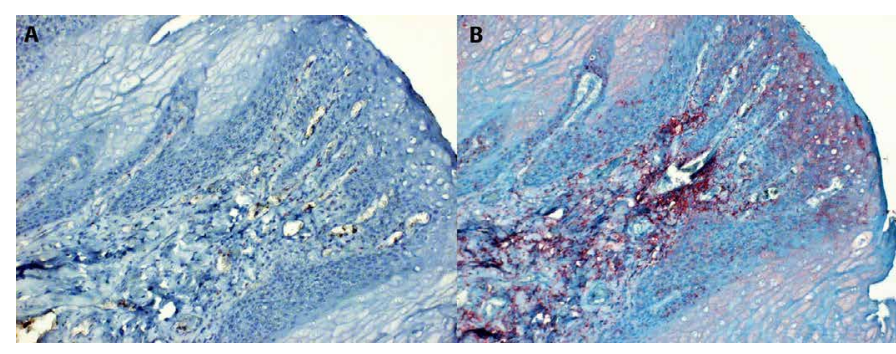

Figure 1. The normal squamous epithelium of the tongue stained for IL-6

1A. A negative parallel section to $1 \mathrm{~B}$ which has been handled similarly, but in the absence of monospecific immune serum (staining control).

1B. Benign proliferation of stratified epithelium at an area adjacent to carcinoma. The IL-6 antigen is present mainly in para-basal location and in the subcutaneous connective tissue (both magn. 120x).

the normal ciliary epithelium lining the mucosa of nasal cavity at least in 1 out of the 3 samples examined. The control staining procedure (in the absence of immune serum) yielded clearly negative results. It should be mentioned that the occasional non-specific binding of antiIL-6 antibody to the intercellular cartilage substance was difficult to interprete.

\section{The presence of IL-6 antigen in laryngeal carcinoma}

Sections from the total of 33 laryngeal carcinoma samples of the tissue array (along with 5 normal laryngeal samples mentioned above), were first evaluated at standard HE is staining and then the results obtained following the anti-IL- 6 antibody treatment were assessed. For the latter, at least 3 parallel sections were compared, namely 2 sections stained with the specific anti-IL-6 serum (i.e. two alternative staining procedures were performed at slightly different $\mathrm{pH}$, for details see Materials and Methods), and in addition, 1 section treated with all the reagents but in the absence of the serum (i.e. negative staining control). As shown on Table 1, all the larynx carcinoma specimens were positive for IL-6 antigen, though their staining intensity ranged from faint to bright.

As the histological grading concerns, 19 samples were classified as conventional (epidermoid) squamous carcinomas (SCC) of grade I being characterized by the presence of keratinized epithelium pearls (Figure 2). In such differentiated carcinomas the IL-6 antigen was present especially in the prickle cells at parabasal locations and in the basal epithelium cells at the basement membrane lining the border to the connective tissue stroma. Furthermore, groups of fibroblasts in the stroma of epidermoid SCCs seemed to show positive cytoplasmic staining. In some of these tumours, groups of a few tumour cells could be identified within the lumen of small veins when revealing a fine granular IL-6 staining. Several of the grade I SCCs might occasionally show less differentiated areas (Figure 2) consisting of groups of irregularly shaped (mainly elongated) cells. In such areas, the IL-6 antigen was concentrated either at the rim of the cytoplasm of prickle cells or at their outer surface, so that the intercellular bridges seemed looking positive.

The rest laryngeal carcinoma cases, however, were low differentiated tumours of grade II and/or poorly differentiated carcinomas of grade III (i.e. altogether 14 undifferentiated SCC cases were found). An example of the latter is depicted on Figure 2, showing rather fine granular staining of irregularly shaped carcinoma cells in combination with extensive positivity of stromal tissue. The Figure 2 demonstrates also an another example of undifferentiated SCC (grade III), which consists of irregular carcinoma cells revealing enlarged nuclei varying in diameter and uneven in size. The cytoplasm of 


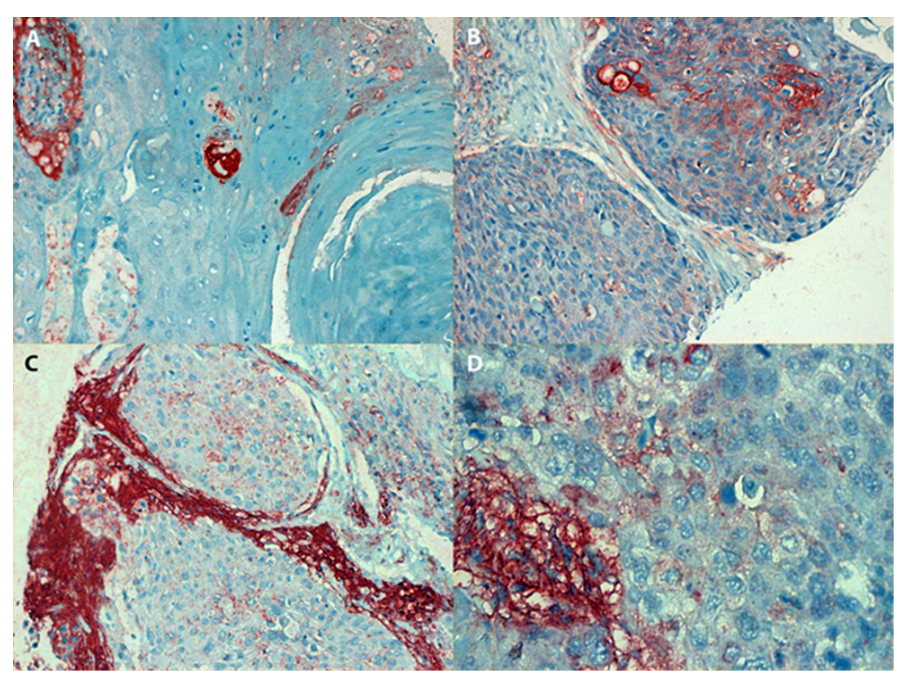

Figure 2. Positive staining of IL-6 antigen in laryngeal carcinoma

1A (in the left, line above): Conventional (epidermoid) squamous carcinoma (grade I) consists of dysplastic cells arranged into typical keratinising whorls. The IL-6 antigen which can be seen in the cytoplasm of prickle cells is either confluent or fine granular, magn. 240x.

2B (in the right, line above): Moderately differentiated area of conventional SCC consists of bands of irregularly shaped tumour cells separated by connective tissue strands. The IL-6 antigen is expressed at the rim of a portion of prickle cells, showing positive staining of intercellular bridges, magn. 240x.

2C (in the left, line below): The undifferentiated SCC of grade II (at growth stage IVA) consists of irregular tumour cells. Fine granular IL-6 antigen can be seen in their cytoplasm, while the stroma reveals extensive extracellular antigen deposits (magn. 240x).

2D (in the right, line below): detail of an undifferentiated SCC grade III (at growth stage IVA). The cytoplasm of anaplastic carcinoma cells contains fine dots of IL-6 antigen; in contrast, abundant extracellular deposits are present in the tumour stroma, magn. $480 \mathrm{x}$

anaplastic cells showed irregular IL-6 staining intensity, namely from faint granular to strongly confluent. In the latter, the anti-IL-6 serum was seen to interact with intercellular connections (desmosomes), in addition to extensive staining of the extracellular antigen deposits (in the tumour stroma).

\section{Expression of IL-6 antigen in tongue carcinoma}

Sections from 21 tongue SCC samples present on the abovementioned tissue array (HN801a) were alternatively stained by the $\mathrm{HE}$ procedure. In contrast, the sections of 34 HNSCC samples obtained from the collection of the Department of Pathology, St. Elisabeth Oncology Hospital in Bratislava, were stained not only with the antiIL-6 specific serum, but also with specific antibody to cytokeratin $5 / 6$ (CK5/6) and by the Masson's trichrome stain (summarized in Table 1). In the former, altogether 9 differentiated carcinomas of grade I were found. The Figure 3 shows a case of conventional SCC grade I tongue tumour revealing squamous cells arranged into epithelial whorls; in this particular tumour, the IL- 6 antigen could be seen mainly in the cytoplasm of dysplastic cells, which were located in parabasal layer, especially in the areas of proliferation growing into the underlying stroma and resembling finger like protrusions. In such cases, the fibroblasts in surrounding stroma were strongly positive along with the abundant extracellular IL-6 antigen deposits. In another differentiated carcinoma sample (classified as verrucous carcinoma, Figure 3), some bands of proliferating cells showed dysplastic appearance especially at tumour margin.

The rest of tongue neoplasms were undifferentiated carcinomas grades II and/or III (namely 11 undifferentiated SCCs and 1 adenocarcinoma of the salivary gland). The cytoplasm of poorly differentiated carcinomas contained relatively few granules of IL-6 antigen; the bands of irregular anaplastic cells were usually negative for the IL-6 antigen, while the surrounding stroma showed strongly positive extracellular deposits. Summing up, out of the 21 tongue carcinoma cases, which sections were presented in the microarray, 9 were relatively differentiated SCCs grade I, while the rest (12 cases) were poorly differentiated and/or undifferentiated carcinomas classified as grade II or III, respectively. In the latter, the IL- 6 antigen was predominantly seen within the tumour stroma, while in the former it could be well detected within the dysplastic squamous tumour cells.

Out of the 34 tongue carcinoma samples (coming from the collection of St. Elisabeth hospital in Bratislava), 27 were classified as conventional SCC (including the to 2 diagnosed as verrucous carcinoma, respectively), while the rest of 7 cases were poorly differentiated SCCs. When stained for IL- 6 antigen, this was seen filling in the cytoplasm of dysplastic cells at sites of parabasal dysplastic squamous cell layer (as already described). Figure 3 shows a case of conventional SCC grade I, in which the IL- 6 antigen can be seen mainly in the cytoplasm of parabasal dysplastic cells. Parallel sections of differentiated SCCs stained for CK5/6 antigen showed that the finger-like and/or papillary protrusions of dysplastic cells were interconnected. In contrast, in poorly and/or moderately differentiated SCCs, the exact site of the initiation of invasive growth was difficult to recognize (Figure 3). The anaplastic cells of poorly differentiated carcinomas were predominantly IL-6 negative. Nevertheless, in these, an abundance of IL-6 antigen was present in within the tumour stroma forming extracellular deposits (Figure 3).

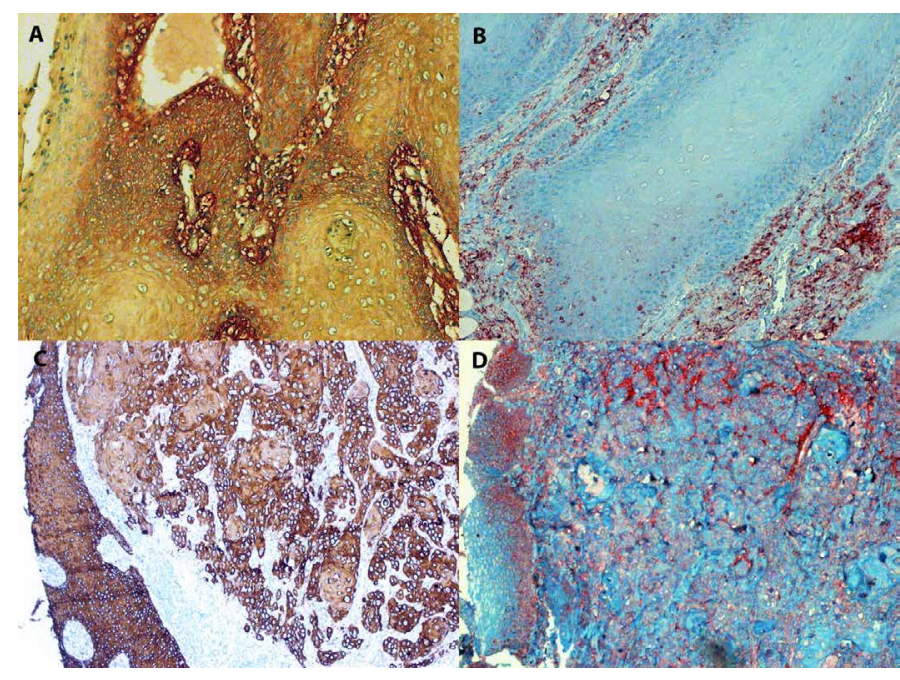

Figure 3. Positive staining for IL-6 in tongue carcinoma

3A (in the left, line above): Conventional SCC (grade I) contains the IL-6 antigen in the cytoplasm of dysplastic parabasal cells as well as in extracellular stroma, magn. 240x.

3B (in the right, line above): Invasive growth of conventional grade I SCC showing granules of IL-6 antigen in the cytoplasm of dysplastic cells at parabasal layer. Staining of the tumour stroma is abundant, magn. $240 \mathrm{x}$.

$3 \mathrm{C} / 3 \mathrm{D}$ (the line below): Two parallel sections of an undifferentiaed SCC stained with the anti-CK5/6 antibody (in the left) and for IL-6 antigen (in the right) document the invasive growth of anaplastic carcinoma cells below the epithelium where dysplastic cells have replaced the original squamous epithelium. While the invasively growing carcinoma cells are mainly negative or stain faint for IL-6, the tumour stroma is strongly positive for extracellular antigen deposits. The dysplastic squamous epithelium cells are positive as well, magn. 120x 


\section{Detection of IL-6 in nasopharyngeal carcinoma}

Together 10 carcinomas coming from the nasopharynx and/or maxillary sinuses were examined, from which 2 were low differentiated SCCs (grade II) of the soft palate, while the rest were poorly differentiated carcinomas (grade III) originating either in the maxillary and/or ethmoid sinuses ( 4 cases) or growing from the nasal cavity (4 cases). The presence of IL- 6 antigen was the most abundant in poorly differentiated carcinomas from nasal cavity. Figure 4 shows the example of a compact tumour invading the submucosa and surrounding tissues, which is in part necrotic. Foci of tumour cells expressing the granular IL- 6 antigen may get occasionally confluent and filling in the cytoplasm, while the extracellular antigen deposits can be hardly recognized. Furthermore, Figure 4 shows an example of undifferentiated nasal carcinoma with very few intraepithelial IL- 6 granules but abundant deposits in the stroma. The poorly differentiated nasal carcinomas, which originated from maxillary sinuses, revealed tumour cells resembling either solid carcinoma or adenocarcinoma. Also the malignant cells invading a vein and/or a lymphatic vessel contained fine granules of IL-6 antigen within cytoplasm.

\section{The presence of the IL-6 antigen in tumours other than SCC}

No IL-6 antigen could be found in pheochromocytoma (a tumour originating from adrenal medulla, (Figure 5). As shown in Table 1, additional 10 tumours other than HNSCC were examined; these were either oat cell carcinomas ( 2 out of 3 were IL- 6 positive) and/or adenocarcinomas, as documented by a sample coming from anorectal area (Figure 5). Carcinomas other than HNSCCs produced IL- 6 by

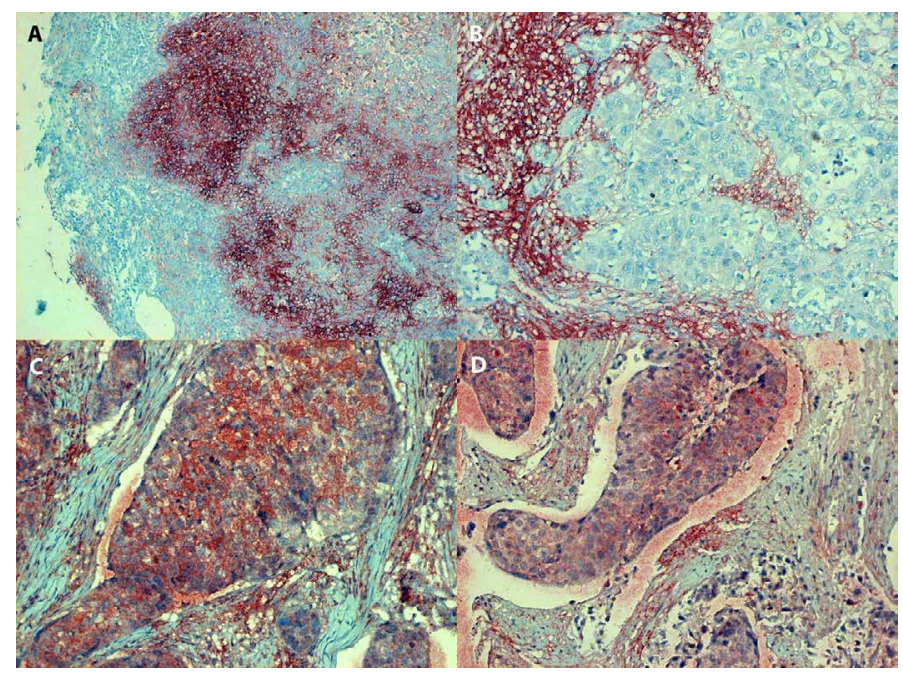

Figure 4. Positive IL-6 staining of nasopharyngeal tumours

4A (in the left, line above): an undifferentiated SCC (grade III/growth stage II) originating from nasal cavity reveals confluent IL-6 positive granules of medium intensity filling in the cytoplasm of poorly differentiated tumour cells; the extracellular deposits in the stroma are difficult to recognize, magn. 120x.

4B (in the right, line above): a similar undifferentiated SCC (grade III/stage II) originating from nasal cavity forms solid and/or glandular structures separated by bands of connective tissue; very few IL-6 positive granules can be seen in the cytoplasm of anaplastic tumour cells. In contrast, the extracellular antigen deposits in the tumour stroma are strongly positive; magn. 240.

4C (in the left, line below): invasive growth of undifferentiated carcinoma originating from maxillary sinus (grade III/stage I) can be seen filling in a lymphatic vessel; several anaplastic tumour cells contain IL- 6 antigen granules, while the extracellular deposits in the stroma are less prominent, magn $240 x$.

4D (in the right, line below): undifferentiated SCC from originating in the maxillary sinus shows invasive growth into a lymphatic vessel; granular staining of the IL-6 antigen can be seen in the cytoplasm of carcinoma cells and occasionally within the tumour stroma, magn. $240 \mathrm{x}$

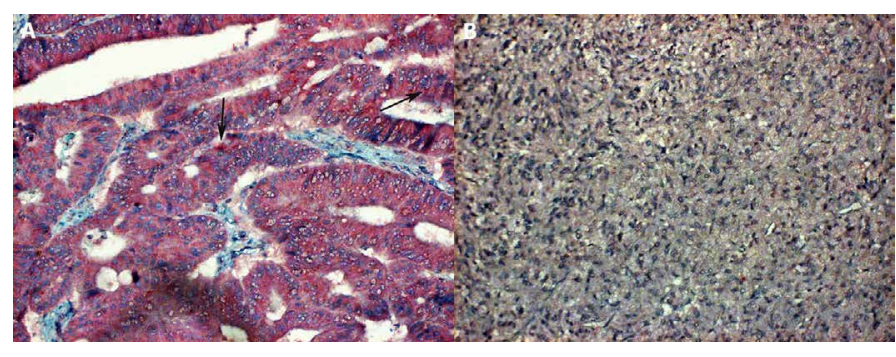

Figure 5. Tumours other than HNSCC

5A. (in the left) : section from biopsy sample nr.16/40248 showing an adenocarcinoma of anorectal area revealing a few faint IL-6 antigen granules (arrows); magn. 240x.

$5 \mathrm{~B}$. (in the right) : the adrenal gland medullar tumour (pheochromocytoma) is completely negative for the IL- 6 antigen, magn. $240 \mathrm{x}$

lower frequency and/or in a few tumour cells. It comes from these data that carcinomas not originating in the head and neck area expressed IL-6 at a lower rate and possibly in less extent.

\section{Discussion}

HNSCC include tumours of larynx, pharynx, oral cavity, tongue and nasal passages, which show a wide range of differentiation (from epidermoid carcinomas to undifferentiated ones). They regularly express cytokines either in vitro (namely the cell lines derived from them) or in vivo, namely secreted into the body fluids of tumour bearing patients. HNSCC is the 6th most common cancer worldwide and poses a significant health problem with more than 45,000 new cases in the United States each year [38]. Among the European countries, the highest incidence of HNSCC is in France followed by high rates noted in Hungary, Slovakia and Slovenia [39,40]. Patients with HNSCC show a poor prognosis since the usual five-year survival rate has been registered among them by a probability of $50-60 \%$ only [41]. The major risk factors for head and neck cancer development are tobacco smoking and alcohol consumption [42]. In addition, the role of human papilloma virus (HPV) has been implicated as a possible cofactor, but the mechanism of action of this virus at HNSCC pathogenesis (in contrast to cervical carcinoma) still not exactly understood [43]. Also, the Epstein-Barr virus (EBV) seems to be somehow involved (at least for the development of SCC at nasopharynx), along with the Torque teno (TTV) and hepatitis C (HCV) viruses, which all may act as possible co-carcinogens increasing the risk of malignant transformation $[44,45]$.

As documented by Di Natale et al. (2011) [46], HNSCC is a typical high cytokine-producing neoplasm, in which IL-6 expression correlates with the tumour aggressiveness. Noteworthy, by using the avidin-biotin-complex $(\mathrm{ABC})$ staining procedure in combination with a specific anti-IL-6 rabbit serum, the distribution of IL-6 immunoreactivity could be demonstrated not only in HNSCCs, but also in many other carcinomas of mammary, colonic, ovarian, and endometrial origin [47]. The cytokines secreted into the neoplastic microenvironment by activated CAFs possibly act on tumour growth by suppressing the immune response as well as by facilitating immune evasion for cancer cells [48]. Especially IL-6 enhances the tumorigenic potential of head and neck cancer stem cells, also termed $\mathrm{ALDH}^{\text {(high)/ }}$ CD44 $4^{\text {(high) }}$ cells [49]. The HNSCC has a small population of uniquely tumorigenic cancer stem cells (CSC) endowed with self-renewal multipotency. Epidermal and dermal cells in active psoriatic plaques from 35 psoriasis patients also stained heavily for IL- 6 as compared other skin diseases as controls [50]. There has been concluded that IL-6 could directly contribute to the epidermal as well as affect the function of dermal inflammatory cells. Certain transcriptional factors have been reported to be involved in the modulation of IL- 6 gene expression 
and have binding sites within the IL- 6 promoter. Namely, in CAFconditioned media, the Janus kinase/signal transducer and activator of transcription (JAK/STAT3) pathway becomes activated. In contrast to normal fibroblasts, in the activated CAFs a considerably higher level of expression of certain cytokine genes, such as for IL-6, CXCL8, tumour necrosis factor (TNF), tumour growth factor (TGFB1) and vascular endothelium growth factor (VEGFA) [51]. Recently, the LIF/IL6-R (Interleukin- 6 receptor) related signalling cascade has been reported getting involved in fibroblast activation. Upon such stimulation, Janus kinase (JAK) is phosphorylated; this further activates STAT3, which is then translocated into the nucleus, where it promotes the transcription of genes responsible for cell growth, differentiation, proliferation and apoptosis [52]. Taken together, the conclusion may be drawn that the abundant expression of IL- 6 antigen within the stroma of HNSCC drives the epithelial squamous cells into more intensive growth when contributing to their dedifferentiation. It has also been suggested that the cytokines produced by cancer cells create optimal growth conditions within the tumour microenvironment, while the cytokines secreted by stromal cells may influence the behaviour of malignant cells.

Transcription induced by the aryl hydrocarbon receptor (AHR) ligand relies on the AHR/ARNT heterodimer and the Brahmarelated gene-1 (BRG1, SMARCA4) binding to the CYP1A1 enhancer region [53]. BRG1 is one of two main catalytic subunits that drive the mammalian SWI/SNF complex; it has been shown to interact with various nuclear receptors and mediate their recruitment to gene promoters. Several authors provided evidence that cetain HNSCC derived cell lines have a constant level of the AHR complex bound to the IL-6 promoter, which may enhance the basal and/or readily inducible IL-6 transcription. Strong-to-moderate IL-6 immunoreactivity was observed in the neoplastic elements present in primary squamous cell carcinomas, in adenocarcinomas of mammary, colonic, ovarian, and endometrial origin, in various adenocarcinomas metastatic to lymph nodes, and in soft tissue tumours including leiomyosarcoma and neurofibrosarcoma. A weak-to-moderate IL-6 immunostaining was observed in Hodgkin's and non-Hodgkin's lymphomas. Classical studies claimed that the most human tumours stain positively for IL-6, adding weight to the hypothesis that IL-6 is a key cytokine that participates in the host-tumour interaction.

Cytokine expression within the tumour microenvironment plays a fundamental role in cancer development and progression since the malignant cells that produce immunosuppressive cytokines can escape the host immune response. In ovarian cancer, several cytokines associated with cellular immunity were correlated to cancer development and patient prognosis, including TNF- $\alpha$, IFN- $\gamma$, IL-6, and corresponding MHC molecules [54]. The higher expression rates of IL- $1 \alpha$ and IL- 6 were also observed in ameloblastomas and related keratocystic tumours suggesting that cytokines in question play a role also in the behaviour odontogenic neoplasms by accelerating bone resorption [55]. The IL- 6 antigen has been demonstrated to be able to induce androgen receptor expression and promote prostate carcinoma progression, thus deemed as a growth factor for most prostate cancer cells in vitro. The findings of Kakourou et al. (2015) [56] provided support for at least a partial positive association between IL- 6 concentrations in plasma and the colon cancer risk, but the same authors observed no similar statistically significant association for rectal cancer. The latter statement seems in accord with our finding of a nearly negative IL-6 expression in rectal adenocarcinoma, however, one must take into account that a single tumour hardly allows to draw a convincing general opinion. Nevertheless, the serum IL-6 level may be considered as an indicator of inflammation and also for promoting carcinogenesis (especially polyposis) in the colon [57]. IL-6 is also a potent stimulator of osteclastogenesis and a sculptor of the tumour microenvironment in the bone marrow of patients with myeloma [58]. The IL-6 was also found overexpressed in the bladder cancer specimens compared with non-malignant tissues at both, the mRNA and protein levels. Transitional cell carcinoma (TCC) of the bladder and positive staining of IL-6 was significantly correlated with worsened clinical stage, higher recurrence and reduced survival rates [59]. Therefore, therapeutic approaches are under development to target STAT3, including molecules that block dimerization or DNA binding by STAT3, or drugs decreasing the STAT3 expression and inhibiting its function [60]. While the EGFR antibody, Cetuximab, has been already approved for HNSCC treatment, additional EGFR-targeting agents are already under trial. These include novel EGFR-specific antibodies, IL-6/ STAT3 antibodies as well as selected tyrosine kinase inhibitors [61-63]. As the latter strategy concerns, however, up-regulated interleukin-6 expression contributes to the development of resistance against the tyrosine kinase inhibitor Erlotinib [64].

Our results showed that HNSCCs revealed intensive staining for IL-6 in tumour stroma, differentiated SCCs (Figure 3). It has been demonstrated that the secretion of this cytokine by TAFs correlates with the worse prognosis of HNSCC due to tumour progression, the development of lymph node metastases and/or invasive growth along with immune evasion [65]. Taken together, our results document the presence production of IL- 6 antigen in the squamous epithelium covering the mucosal surface of mouth cavity, tongue and laryngeal area, but its especially abundant production in SCCs originating from above mentioned tissues.

\section{Acknowledgements}

The authors thank ass. prof. Karol Kajo, MD, $\mathrm{PhD}$, for his cooperative help when providing the anti-CK5/6 serum as well as the "domestic" biopsy samples of tongue and mouth carcinomas coming from the archives of the Pathology Department of St. Elisabeth Oncology Hospital in Bratislava, Slovakia. Cooperation with the University of Szeged was supported by the grant GINOP 2.3.2-15-201600011 provided by the European Regional Dvelopment Fund of EU.

\section{Ethical statement}

The authors declare that all the material examined was coming either from sections purchased by a third party or from the biopsy collection of Pathology Department of above mentioned Oncology hospital, where the tumour tissue was removed at health saving operations and first examined for diagnostic reason, and just later on for the purpose this study.

\section{References}

1. Guo Y, Xu F, Lu T, Duan Z, Zhang Z (1992) Interleukin-6 signaling pathway in targeted therapy for cancer. Cancer Treat Rev 38: 904-910.

2. Kishimoto T (2006) Interleukin-6: discovery of a pleiotropic cytokine. Arthritis Res Ther 8 Suppl 2: S2. [Crossref]

3. Gallo O, Gori AM, Attanasio M, Martini F, Fini-Storchi O, et al. (1992) Interleukin-6 serum level and monocyte production in head and neck cancer. Br J Cancer 65: 479480. [Crossref]

4. Klein JD, Grandis JR (2010) The molecular pathogenesis of head and neck cancer Cancer Biol Ther 9: 1-7. [Crossref]

5. Tabibzadeh SS, Poubouridis D, May LT, Sehgal PB (1989) Interleukin-6 immunoreactivity in human tumors. Am J Pathol 135: 427-433. [Crossref]

6. Takahashi H, Sakakura K, Kawabata-Iwakawa R, Rokudai S, Toyoda M (2015) Immunosuppressive activity of cancer-associated fibroblasts in head and neck squamous cell carcinoma. Cancer Immunol Immunother 64: 1407-17. 
7. Johnston PA, Grandis JR (2011) STAT3 signaling: anticancer strategies and challenges. Mol Interv 11: 18-26. [Crossref]

8. Kanazawa T, Nishino H, Hasegawa M (2007) Interleukin-6 directly influences proliferation and invasion potential of head and neck cancer cells. Eur Arch Otorhinolaryngol 264: 815-821.

9. Kumari N, Dwarakanath BS, Das A, Bhatt AN (2016) Role of interleukin-6 in cancer progression and therapeutic resistance. Tumor Biol 37: 11553-11572.

10. Reznick AZ, Hershkovich O, Nagler RM (2004) Saliva-a pivotal player in the pathogenesis of oropharyngeal cancer. Br J Cancer 91: 111-118. [Crossref]

11. Culig Z (2013) Interleukin-6 as a therapy target in oral squamous carcinoma. Expert Opin Ther Targets 17: 53-59. [Crossref]

12. Frank DA (2007) STAT3 as a central mediator of neoplastic cellular transformation. Cancer Lett 251: 199-210

13. Heinrich PC, Behrmann I, Muller-Newen G, Schaper F, Graeve L (1998) Interleukin-6type cytokine signalling through the gp130/Jak/STAT pathway. Biochem J 334: 297 314.

14. Malamud D (2011) Saliva as a diagnostic fluid. Dent Clin North Am 55: 159-178. [Crossref]

15. Rhodus NL, Ho V, Miller CS, Myers S, Ondrey F (2005) NF- kappaB dependent cytokine levels in saliva of patients with oral preneoplatic lesions and oral squamous cell carcinoma. Cancer Detect Prev 29: 42-45.

16. Sahibzada HA, Khurshid Z, Khan RS, Naseem M, Siddique KM, et al. (2017) Salivary IL-8, IL-6 and TNF- $\alpha$ as potential diagnostic biomarkers for oral cancer. Diagnostics (Basel) 7. [Crossref]

17. Sato J, Ohuchi M, Abe K, Satoh T, Abe T, et al. (2013) Correlation between salivary interleukin-6 levels and early locoregional recurrence in patients with oral squamous cell carcinoma: preliminary study. Head \& Neck 35: 889-894.

18. Seethala RR, Gooding WE, Handler PN (2008) Immunohistochemical analysis of phosphotyrosine signal transducer and activator of transcription 3 and epiderma growth factor receptor autocrine signaling pathways in head and neck cancers and metastatic lymph nodes. Clin Cancer Res 14: 1303-1309.

19. Stanam A, Love-Homan L, Joseph TS, Espinosa-Cotton M, Simons AL (2015) Upregulated interleukin-6 expression contributes to erlotinib resistance in head and neck squamous cell carcinoma. Mol Oncol 9:1371-1383.

20. Ara T, Nakata R, Sheard MA, Shimada H, Buettner R, et al. (2013) Critical role of STAT3 in IL-6-mediated drug resistance in human neuroblastoma. Cancer Res 73: 3852-3864. [Crossref]

21. Brocke-Heidrich K, Kretzschmar AK, Pfeifer G (2004) Interleukin-6-dependent gene expression profiles in multiple myeloma INA-6 cells reveal a Bcl-2 family independent survival pathway closely associated with Stat3 activation. Blood 103: 242-251

22. Hong D, Angelo LS, Kurzrock R (2007) Interleukin-6 and its receptor in cancer: implications for translational therapeutics. Cancer 110: 1911-1928

23. Bose P, Brockton NT, Dort JC (2013) Head and neck cancer: from anatomy to biology. Int J Cancer 133: 2013-2023. [Crossref]

24. Sharma M, Bairy I, Pai K, Satyamoorthy K, Prasad S, et al. (2011) Salivary IL-6 levels in oral leukoplakia with dysplasia and its clinical relevance to tobacco habits and periodontitis. Clin Oral Investig 15: 705-714. [Crossref]

25. Korostoff A, Reder L, Masood R, Sinha UK (2011) The role of salivary cytokine biomarkers in tongue cancer invasion and mortality. Oral Oncology 47: 282-287.

26. Andersson BÅ, Lewin F, Lundgren J, Nilsson M, Rutqvist LE, et al. (2014) Plasma tumor necrosis factor- $\alpha$ and $C$-reactive protein as biomarker for survival in head and neck squamous cell carcinoma. J Cancer Res Clin Oncol 140: 515-519.

27. Duffy SA, Taylor JM, Terrell JE, Islam M, Li Y, et al. (2008) Interleukin-6 predicts recurrence and survival among head and neck cancer patients. Cancer 113: 750-757. [Crossref]

28. Katakura A, Kamijama I, Takano N, Shibahara T, Muramatsu T, et al. (2007) Comparison of salivary cytokine levels in oral cancer patients and healthy subjects. Bull Tokyo Dent Coll 48: 199-203.

29. Brailo V, Vucicevic-Boras V, Cekic-Arambasim A, Alajbeg IZ, Milenovic A, et al. (2006) The significance of salivary interleukin 6 and tumor necrosis factor alpha in patients with oral leukoplakia and oral cancer. Oral Oncology 42: 370-373.

30. Dineshkumar T, Ashwini BK, Rameshkumar A, Rajashree P, Ramya R, et al. (2016) Salivary and serum Interleukin-6 Levels in oral premalignant disorders and squamous cell carcinoma: diagnostic value and clinicopathologic correlations. Asian Pac J Cancer Prev 17: 4899-4906.
31. Javaid MA, Ahmed AS, Durand R, Tran SD (2016) Saliva as a diagnostic tool for oral and systemic diseases. J Oral Biol Craniofac Res 6: 66-75. [Crossref]

32. Sato J, Ohuchi M, Abe K, Satoh T, Abe T, et al. (2013) Correlation between salivary interleukin-6 levels and early locoregional recurrence in patients with oral squamous cell carcinoma: preliminary study. Head \& Neck 35: 889-894.

33. Juretic M, Cerovic R, Belušic-Gobic M, Brekalo Pršo I, Kqiku L, et al. (2013) Salivary levels of TNF- $\alpha$ and IL-6 in patients with oral premalignant and malignant lesions. Folia Biol (Praha) 59: 99-102.

34. Aderhold C, Grobschmidt GM, Sauter A, Faber A, Hörmann K, et al. (2014) Interleukin 4, interleukin 6 and osteopontin-serological markers of head and neck malignancy in primary diagnostics: A pilot study. Oncol Lett 8: 1112-1118.

35. Hunter CA, Jones SA (2015) IL-6 as a keystone cytokine in health and disease. Nat Immunol 16: 448-457. [Crossref]

36. Panneer Selvam N, Sadaksharam J (2015) Salivary interleukin-6 in the detection of oral cancer and precancer. Asia Pac J Clin Oncol 11: 236-241. [Crossref]

37. Sriuranpong V, Park JI, Amornphimoltham P, Patel V, Nelkin BD, et al. (2003) Epidermal growth factor receptor-independent constitutive activation of STAT3 in head and neck squamous cell carcinoma is mediated by the autocrine/paracrine stimulation of the interleukin 6/gp130 cytokine system. Cancer Res 63: 2948-2956.

38. Jemal A, Siegel R, Ward E, Murray T, Xu J, et al. (2007) Cancer statistics. CA Cancer J Clin 57: 43-66. [Crossref]

39. Tanaka T, Narazaki M, Kishimoto T (2014) IL-6 in inflammation, immunity, and disease. Cold Spring Harb Perspect Biol 6: a016295. [Crossref]

40. Vigneswaran N, Williams MD (2014) Epidemiologic trends in head and neck cancer and aids in diagnosis. Oral Maxillofac Surg Clin North Am 26: 123-141. [Crossref]

41. Islam M, Sharma S, Teknos TN (2014) RhoC regulates cancer stem cells in head and neck squamous cell carcinoma by overexpressing IL-6 and phosphorylation of STAT3. PLoS One 9: e88527. [Crossref]

42. Sharma M, Bairy I, Pai K, Satyamoorthy K, Prasad S, et al. (2011) Salivary IL-6 levels in oral leukoplakia with dysplasia and its clinical relevance to tobacco habits and periodontitis. Clin Oral Investig 15: 705-714. [Crossref]

43. Shaw R, Beasley N (2016) Aetiology and risk factors for head and neck cancer: United Kingdom National Multidisciplinary Guidelines, J Laryngol Otol 130: S9-S12.

44. Hettmann A, Demcsák A, Decsi G, Bach Á, Pálinkó D, et al. (2016) Infectious Agents Associated with Head and Neck Carcinomas. Adv Exp Med Biol 897: 63-80. [Crossref]

45. Hettmann A, Demcsák A, Bach Á, Decsi G, Dencs Á et al. (2016) Detection and phylogenetic analysis of torque teno virus in salivary and tumor biopsy samples from head and neck carcinoma patients. Intervirology 59: 123-129.

46. Di Natale BC, Jennifer C Schroeder, Gary H Perdew (2011) Ah receptor antagonism inhibits constitutive and cytokine inducible IL6 production in head and neck tumor cell lines. Mol Carcinog 50: 173-183.

47. Stanam A, Love-Homan L, Joseph TS, Espinosa-Cotton M, Simons AL (2015) Upregulated interleukin-6 expression contributes to erlotinib resistance in head and neck squamous cell carcinoma. Mol Oncol 9: 1371-1383.

48. Routray S, Sunkavali A, Bari KA (2014) Carcinoma-associated fibroblasts, its implication in head and neck squamous cell carcinoma: a mini review. Oral Dis 20: 246-253. [Crossref]

49. Finkel KA, Warner KA, Kerk S, Bradford CR, McLean SA, et al. (2016) IL-6 inhibition with MEDI5117 decreases the fraction of head and neck cancer stem cells and prevents tumor recurrence. Neoplasia 18: 273-281. [Crossref]

50. Grossman RM, Krueger J, Yourish D, Granelli-Piperno A, Murphy DP et al. (1989) Interleukin 6 is expressed in high levels in psoriatic skin and stimulates proliferation of cultured human keratinocytes. Proc Natn Acad Sci 86: 6367-6371.

51. Stark J, Li H, Kraft P (2009) Circulating prediagnostic interleukin-6 and C-reactive protein and prostate cancer incidence and mortality. Int J Cancer 124: 2683-2689.

52. Kuzet SE, Gaggioli C (2016) Fibroblast activation in cancer: when seed fertilizes soil. Cell Tissue Res 365: 607-619. [Crossref]

53. Vazquez MI, Catalan-Dibene J, Zlotnik A (2015) B cell responses and cytokine production are regulated by their immune microenvironment. Cytokine 74: 318-326.

54. Wang S, Hankinson O (2002) Functional involvement of the Brahma/SWI2-related gene 1 protein in cytochrome P4501A1 transcription mediated by the aryl hydrocarbon receptor complex. J Biol Chem 277: 11821-11827. 
55. Sengüven B, Oygür T (2011) Investigation of interleukin-1 alpha and interleukin-6 expression and interleukin-1 alpha gene polymorphism in keratocystic odontogenic tumors and ameloblastomas. Med Oral Patol Oral Cir Bucal 16: e467-472. [Crossref]

56. Kakourou A, Koutsioumpa C, Lope DS, Hoffman-Bolton J, Bradwin G, et al. (2015) Androgen receptor signaling: mechanism of interleukin-6 inhibition. Cancer Res 64 2619-2626.

57. Bobe G, Albert PS, Leah B. Sansbury LB, Elaine Lanza E, et al. (2010) Interleukin-6 as a potential indicator for prevention of high-risk adenoma recurrence by dietary flavonols in the polyp prevention trial. Cancer Prev Res 3: 764-775.

58. Rosean TR, Tompkins van S, Tricot, G, Holman CJ, Olivier AK, et al. (2014) Preclinical validation of interleukin 6 as a therapeutic target in multiple myeloma. Immunol Res 59: $188-202$.

59. Chen MF, Lin PY, Wu ChF, Chen WC, Chun TW (2013) IL-6 expression regulates tumorigenicity and correlates with prognosis in bladder cancer. Plos One 8: e61901.
60. Leeman RJ, Lui VW, Grandis JR (2006) STAT3 as a therapeutic target in head and neck cancer. Expert Opin Biol Ther 6: 231-241. [Crossref]

61. Kitamura H, Ohno Y, Toyoshima Y, Ohtake J, Homma S, et al. (2017) Interleukin-6/ STAT3 signaling as a promising target to improve the efficacy of cancer immunotherapy. Cancer Sci 108: 1947-1952.

62. Leeman Neill RJ, Wheeler SE, Singh SV (2009) Guggulsterone enhances head and neck cancer therapies via inhibition of signal transducer and activator of transcription-3. Carcinogenesis 30: 1848-1856.

63. Liu Q, Yu S, Li A, Xu H, Han X, et al. (2017) Targeting interlukin-6 to relieve immunosuppression in tumor microenvironment. Tumour Biol 39: 1-1.

64. Shaw R, Beasley N (2016) Aetiology and risk factors for head and neck cancer: United Kingdom National Multidisciplinary Guidelines, J Laryngol Otol 130: S9-S12.

65. Leef G, Thomas SM (2013) Molecular communication between tumor-associated fibroblasts and head and neck squamous cell carcinoma. Oral Oncol 49: 381-386.

Copyright: (2019 Rajčáni J. This is an open-access article distributed under the terms of the Creative Commons Attribution License, which permits unrestricted use, distribution, and reproduction in any medium, provided the original author and source are credited. 